\title{
Effect of exogenous nitric oxide on sperm motility in vitro
}

Jiangtao Wang ${ }^{1 \dagger}$, Qingliu He ${ }^{2 \dagger}$, Xingyu Yan ${ }^{2 *}$, Youmei $\mathrm{Cai}^{3}$ and Junyi Chen ${ }^{2}$

\begin{abstract}
Background: Nitric oxide (NO) has been shown to be important in sperm function, and the concentration of NO appears to determine these effects. Studies have demonstrated both positive and negative effects of NO on sperm function, but have not been able to provide a clear link between NO concentration and the extent of exposure to NO. To study the relationship between nitric oxide and sperm capacitation in vitro, and to provide a theoretical basis for the use of NO-related preparations in improving sperm motility for in vitro fertilization, we investigated the effects of $\mathrm{NO}$ concentration and time duration at these concentrations on in vitro sperm capacitation in both normal and abnormal sperm groups. We manipulated NO concentrations and the time duration of these concentrations using sodium nitroprusside (an NO donor) and NG-monomethyl-L-argenine (an NO synthase inhibitor).

Results: Compared to the normal sperm group, the abnormal sperm group had a longer basal time to reach the appropriate concentration of $\mathrm{NO}(p<0.001)$, and the duration of time at this concentration was longer for the abnormal sperm group $(p<0.001)$. Both the basal time and the duration of time were significantly correlated with sperm viability and percentage of progressive sperm $(p<0.001)$. The experimental group had a significantly higher percentage of progressive sperm than the control group $(p<0.001)$.

Conclusions: We hypothesize that there is a certain regularity to both $\mathrm{NO}$ concentration and its duration of time in regards to sperm capacitation, and that an adequate duration of time at the appropriate NO concentration is beneficial to sperm motility.
\end{abstract}

Keywords: Nitric oxide, Sperm capacitation, Sperm motility, Time, Human

\section{Background}

Infertility is a common clinical problem, affecting approximately $15 \%$ of couples, with a male factor influence in $30-60 \%$ of cases [1]. The effects of reactive oxygen species (ROS) and their importance in both physiological and pathophysiological events has been the subject of considerable study in recent years. Several ROS, including hydrogen peroxide $\left(\mathrm{H}_{2} \mathrm{O}_{2}\right)$, superoxide anion, and nitric oxide (NO), have been shown to be involved in processes important to sperm physiology. Under normal, tightly regulated physiologic conditions, these ROS are essential to in vitro events necessary for the fertilizing ability of sperm [2]. Nitric oxide plays an important role in a variety of physiologic processes, including cellular information

\footnotetext{
* Correspondence: xingyuyan61@163.com

${ }^{\dagger}$ Equal contributors

${ }^{2}$ Departments of Urology, Second Affiliated Hospital, Fujian Medical

University, Quanzhou, Fujian, China

Full list of author information is available at the end of the article
}

transmission [3], cellular defense [4], and as a regulator in both male and female reproductive functions [5]. The majority of evidence supports the view that at levels exceeding physiologic concentrations (generally considered to be less than one micromolar [6]), disruption of sperm function occurs, but that at low levels, $\mathrm{NO}$ is essential for sperm function. At physiologic levels, NO has been shown to be important in sperm capacitation [7-11] and acrosome reaction [8,11,12], in the maintenance of sperm motility [13], and may have an anti-apoptotic effect in sperm [11].

While some investigators have found no evidence supporting a detrimental effect of NO on sperm [14], the majority of evidence supports the notion that supraphysiologic concentrations of NO negatively affect sperm function. Salvolini et al. [15] provided evidence that increased nitric oxide synthase (NOS) activity and elevated tyrosine nitration may be contributory to the pathogenesis of idiopathic asthenozoospermia. A study by Weinberg 
et al. [16] showed that increased NO from addition of sodium nitroprusside (SNP, an NO donor), inhibited sperm motility and was correlated with NO-mediated inhibition of sperm cellular respiration. Other studies have provided evidence that elevated levels of $\mathrm{NO}$ decrease motility [17-23], usually in a concentration dependent $[17,18]$ and time dependent [18] manner, and are associated with increased sperm toxicity [17] and apoptosis [20].

However, although the physiologic effects on sperm modulated by $\mathrm{NO}$ appear to depend on both the concentration of NO and the duration of NO exposure [24], further clarification is needed regarding the relationship between the timing of $\mathrm{NO}$ exposure and the concentration of NO and sperm motility and capacitation. The present study collected both normal and abnormal semen samples for real-time monitoring of both NO concentration and time changes during sperm capacitation. Changes in these parameters were also monitored while artificially controlling the NO concentration with the addition of SNP and NG-monomethyl-L-arginine (L-NMMA, an NOS inhibitor), which allowed us to hypothesize on the relationship between NO concentration, time changes, sperm motility and sperm capacitation.

\section{Results}

In the normal sperm group, the basal time (T1) needed to reach the appropriate NO concentration for capacitation was $32.09 \pm 4.90$ minutes. This concentration of nitric oxide (D) was $19912.33 \pm 1359.95 \mathrm{nM}$, and the duration of time (T2) at this concentration of $\mathrm{NO}$ was $11.27 \pm$ 2.42 minutes. In the abnormal sperm group, $\mathrm{T} 1$ was $79.46 \pm 9.61$ minutes, $\mathrm{D}$ was $19513.60 \pm 1914.72 \mathrm{nM}$, and T2 was $31.89 \pm 4.92$ minutes.

Based on the result of NO concentration over time during in vitro sperm capacitation in both the normal and abnormal semen groups, we reached the preliminary conclusion that, compared with the normal sperm group, the abnormal sperm group took longer to reach an appropriate NO concentration $(\mathrm{t}=-17.017, p<0.001)$, and had a longer duration at these concentrations $(\mathrm{t}=-14.582, p<0.001)$, but otherwise had no significant differences at these concentrations $(\mathrm{t}=0.658, p=0.516>$ 0.05). These data are shown in Figures 1, 2 and 3.

The T1s of the normal and abnormal sperm groups were significantly correlated with sperm viability $(\mathrm{r}=-0.888$, $p<0.001)$ and the percentage of progressive sperm $(\mathrm{r}=-0.952, p<0.001)$. The T2s of the normal and abnormal sperm groups were significantly correlated with sperm viability $(\mathrm{r}=-0.853, p<0.001)$ and the percentage of progressive sperm $(\mathrm{r}=-0.942, p<0.001)$. The NO concentration (D) of both groups had no obvious correlation with sperm viability $(\mathrm{r}=0.113, p=0.554>$ $0.05)$ and percentage of progressive sperm $(r=0.120$,

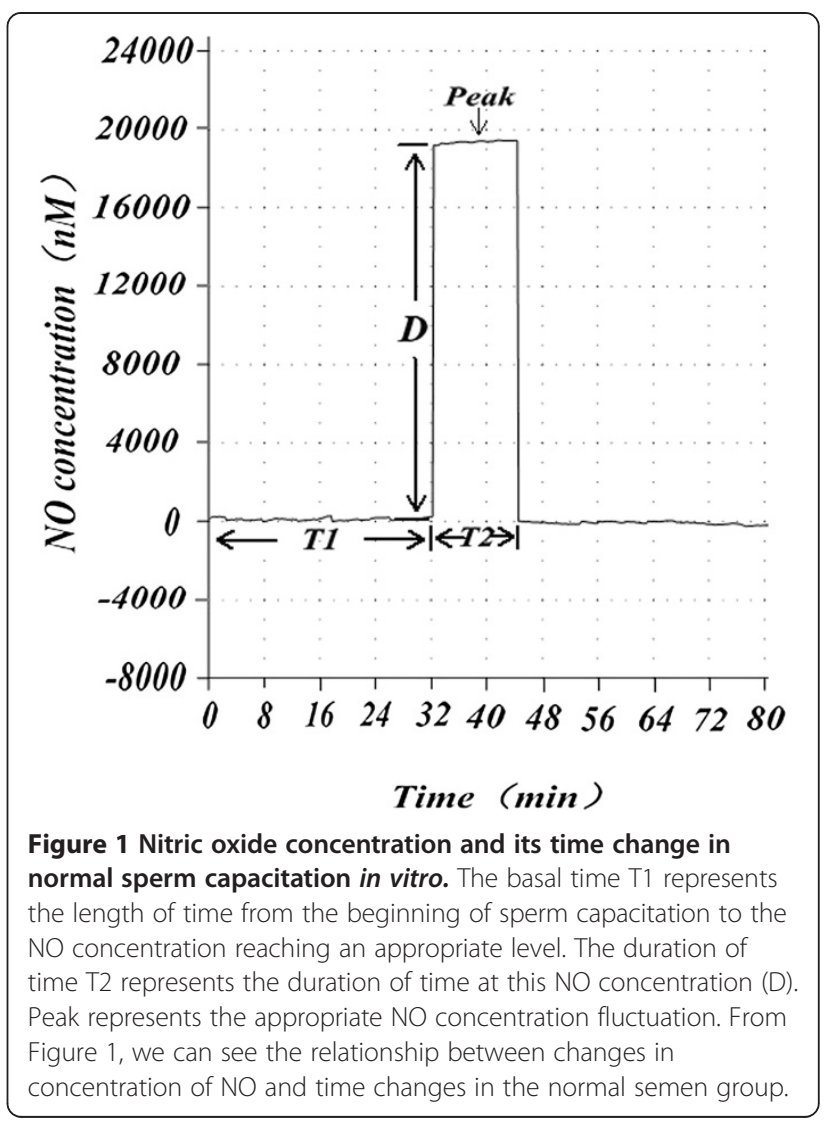

$p=0.528>0.05)$. Compared with the normal sperm group, the abnormal sperm group had lower sperm viability $(\mathrm{t}=15.598, p<0.001)$ and a lower percentage of progressive sperm $(\mathrm{t}=24.003, p<0.001)$. These data are shown in Figures 4 and 5.

For the next part of the experiment, T1s of the control group (C1) and experimental group (C2) were $78.69 \pm$ 8.92 minutes and $79.21 \pm 9.12$ minutes, respectively. Compared with $\mathrm{C} 1, \mathrm{C} 2$ showed no significant difference in T1. In the experimental group C2, SNP (100 nmol/L) was continuously added, beginning at thirty minutes after starting measurement of NO (the average time of normal sperm capacitation in vitro). There was an immediate increase in $\mathrm{NO}$ concentration, equivalent to the $\mathrm{T} 1$ peak in advance seen in Figure 6. This NO concentration was maintained by regulating the addition of SNP. When L-NMMA (10 mmol/L) was continuously added to C2, starting at forty-one minutes after starting measurement of NO (the point of average duration of normal sperm capacitation in vitro), the NO concentration rapidly returned to baseline levels, equivalent to T2 levels shorten. This NO concentration and time change curve is similar to the one seen in the normal semen group.

By analyzing sperm quality parameters of the control and experimental groups, we found that the sperm viability in C1 was $40.92 \pm 1.53 \%$, the percentage of progressive 


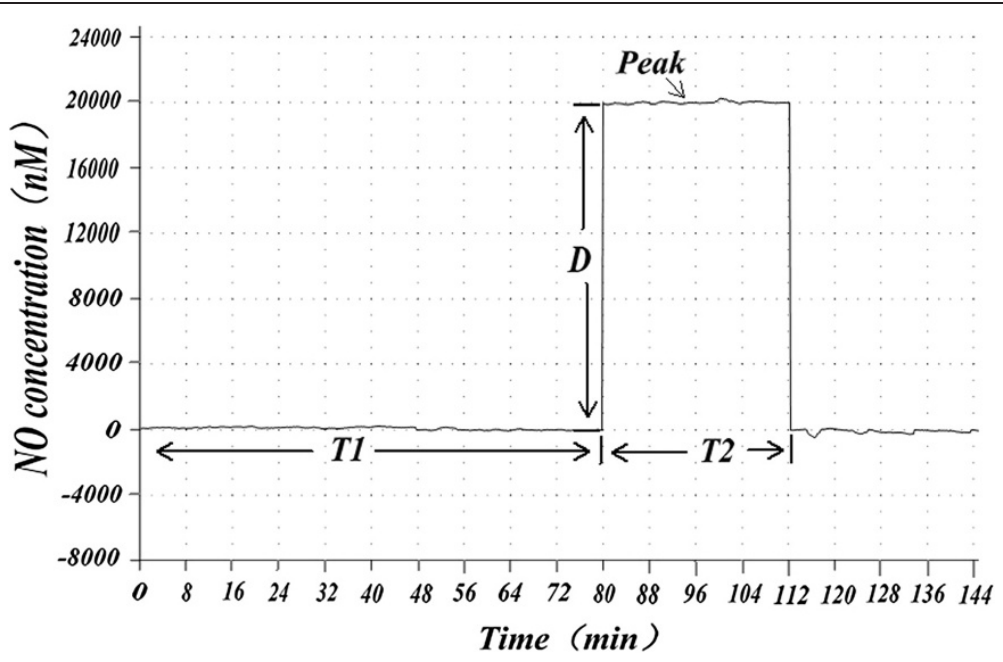

Figure 2 Nitric oxide concentration and its time change in abnormal sperm capacitation in vitro. $T 1, T 2$, and D as in Figure 1. In Figure 2, both $\mathrm{T} 1$ and $\mathrm{T} 2$ were longer in abnormal sperm than in normal sperm.

sperm in $\mathrm{C} 1$ was $11.73 \pm 0.88 \%$, the sperm viability in $\mathrm{C} 2$ was $39.35 \pm 1.43 \%$, the percentage of progressive sperm in $\mathrm{C} 2$ was $21.00 \pm 1.21 \%$. The experimental group had a significant increase in percentage of progressive sperm $(\mathrm{t}=-6.201, p<0.001)$, while both groups had no obvious difference in sperm viability $(\mathrm{t}=0.746, p=0.426>0.05)$. These data are shown in Figure 7.

\section{Discussion}

The current evidence in the medical literature shows that ROS, including NO, are important in sperm function
$[5,25,26]$, and that, in the case of $\mathrm{NO}$, the concentration appears to determine the effects on sperm motility [5]. As discussed earlier, studies have demonstrated both positive and negative effects of $\mathrm{NO}$ on sperm function, but have not been able to provide a clear link between NO concentration and the extent of exposure to NO.

Our present study sought to address this and was based on a continuous measurement of direct induction and manipulation of $\mathrm{NO}$ and observation of the time duration of NO concentration. Through real-time monitoring of the $\mathrm{NO}$ concentration change during sperm

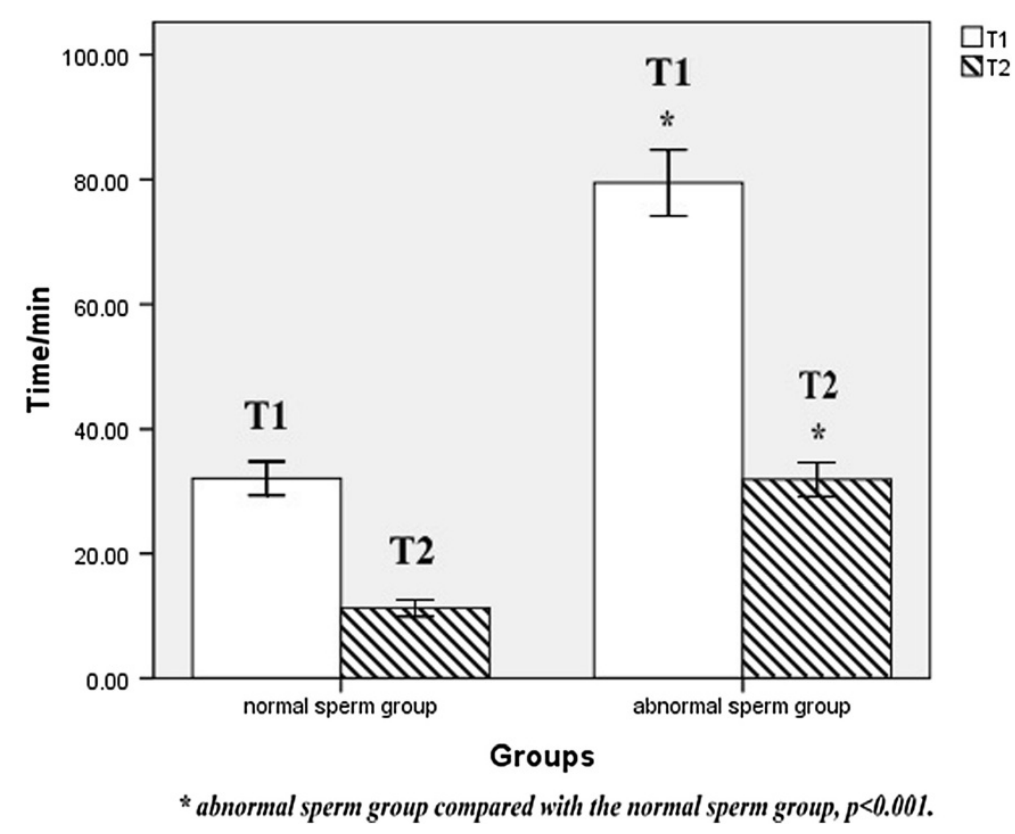

Figure 3 The basal time (T1) and the duration of time (T2) in normal and abnormal sperm groups. Results were expressed as mean \pm standard deviation, $\mathrm{n}=15$. Both $\mathrm{T} 1$ and $\mathrm{T} 2$ of the abnormal sperm group were longer $(p<0.001)$ than that of the normal sperm group. 


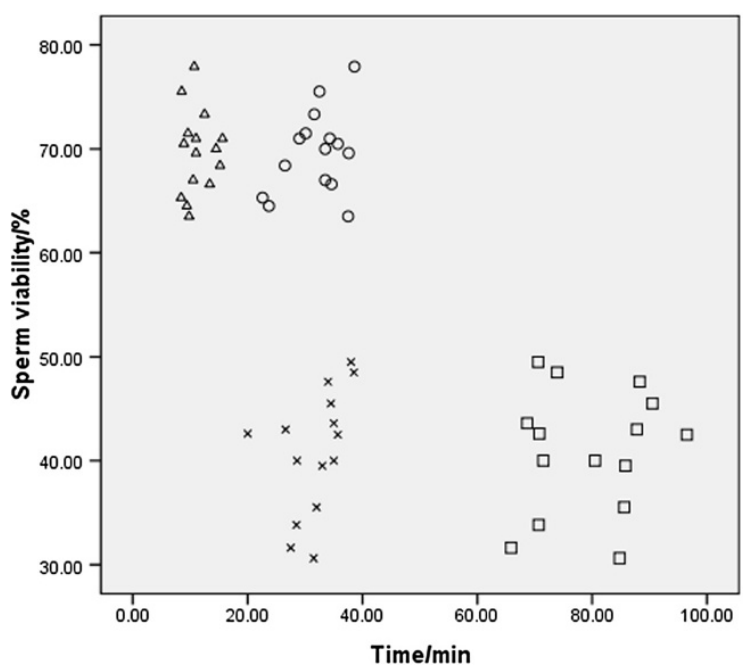

OT1 of normal sperm group

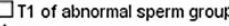
$\Delta T 2$ of normal sperm group $\times T 2$ of abnormal sperm group

Figure 4 The relationship between sperm viability and time in normal and abnormal sperm groups. In this figure, the $Y$-axis represents the percentage of viable sperm in all samples and the $X$-axis represents the time (T1 or T2) in the normal and abnormal sperm groups. The correlation between sperm viability and $\mathrm{T} 1(\mathrm{r}=-0.888, p<0.001, \mathrm{n}=15)$ and between sperm viability and $\mathrm{T} 2(\mathrm{r}=-0.853, p<0.001, \mathrm{n}=15)$ was established through Pearson correlation analysis. Using the independent sample t-test, sperm viability in the abnormal sperm group was obviously lower than the normal sperm group $(\mathrm{t}=15.598, \mathrm{p}<0.001, \mathrm{n}=15)$.

capacitation in vitro, our current experiment provides empirical evidence furthering the understanding of the relationship between sperm capacitation in vitro, sperm mobility, NO concentration and time changes. Compared to the normal semen group, the abnormal semen group had a longer basal time before reaching an appropriate
$\mathrm{NO}$ concentration and remained at this concentration for a longer time. These time parameters were significantly correlated with both sperm viability and the percentage of progressive sperm. Elevated concentrations of NO-related enzymes (protein kinase A) can be detected 30 minutes after the initiation of sperm capacitation in vitro, suggesting

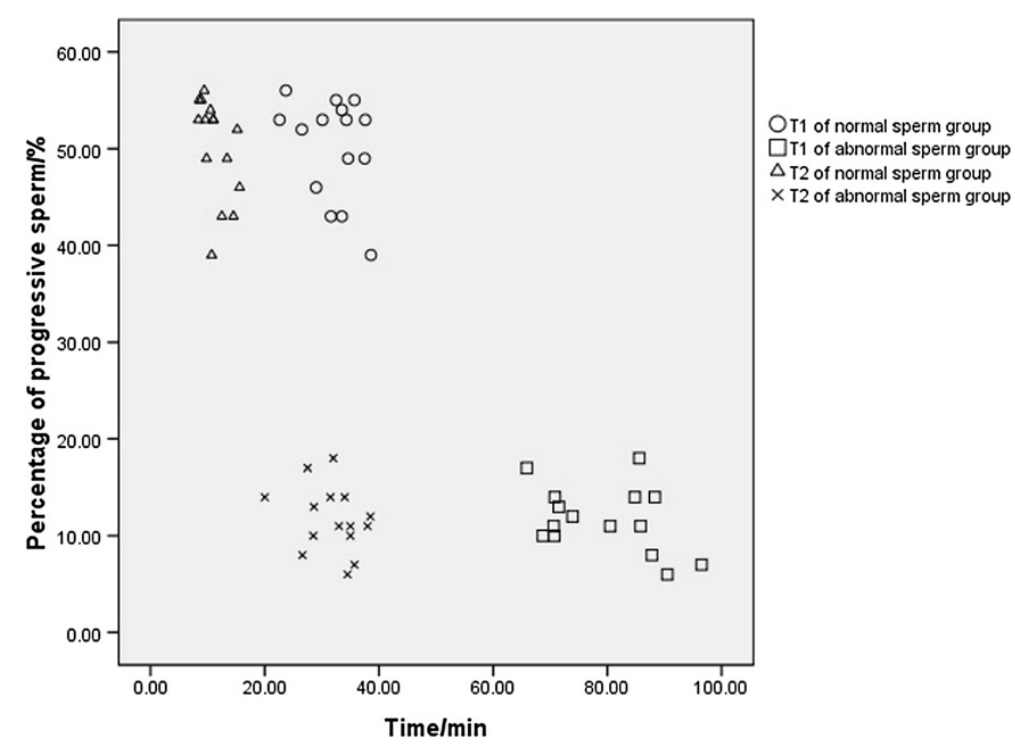

Figure 5 The relationship between percentage of progressive sperm and time in normal and abnormal sperm groups. In this figure, the $Y$-axis represents the percentage of progressive sperm in all samples and the X-axis represents the time (T1 or T2) in the normal and abnormal sperm groups. The correlation between the percentage of progressive sperm and $T 1(r=-0.952, p<0.001, n=15)$ and between the percentage of progressive sperm and T2 $(r=-0.942, p<0.001, n=15)$ was established through Pearson's correlation analysis. Using the independent sample $t$-test, the percentage of progressive sperm in the abnormal sperm group was obviously lower than the normal sperm group ( $t=24.003$, $p<0.001, \mathrm{n}=15)$. 


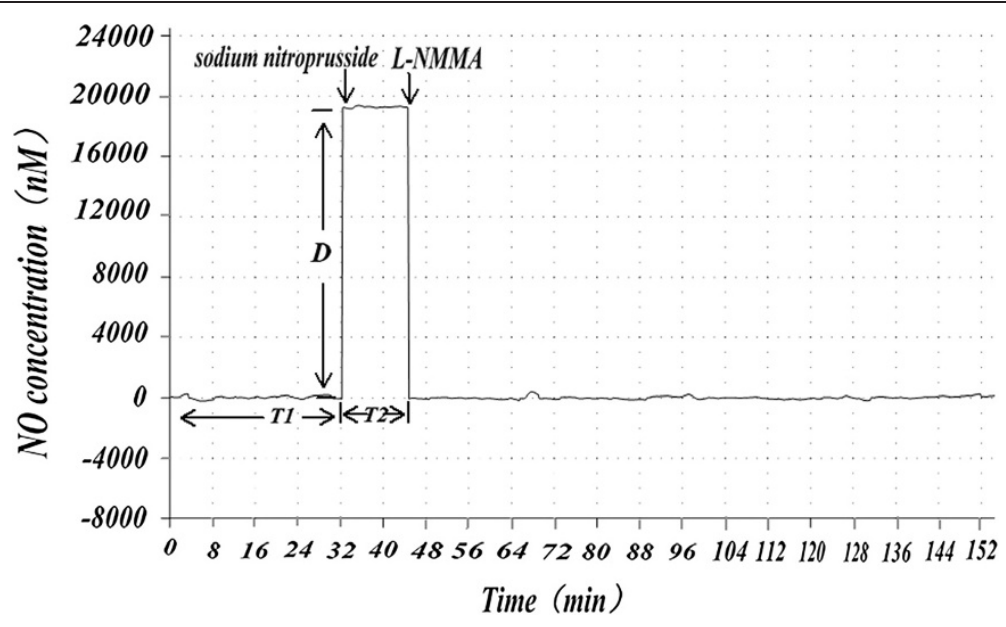

Figure 6 The experimental group's NO concentration and time change curve detected after adding SNP and L-NMMA. T1, T2, and D as in Figure 1. SNP and L-NMMA were used to manipulate NO concentration in the experimental group, and, as seen in Figure 6, the experimental group's NO concentration and time change curve is very similar to that of the normal semen group.

that the change of $\mathrm{NO}$ concentration is most active at this point [27]. This most active time is approximately the same as the T1 of our normal semen group. An extended T1 in the abnormal semen group, seen as a time delay in the change of NO concentration reaching its most active point, could influence sperm viability and motility.

In addition, there were no obvious differences in the relative degree of $\mathrm{NO}$ concentration in both groups, and no significant correlation with sperm motility or other sperm quality parameters. Our findings support the assertion that sperm quality, particularly the percentage of progressive sperm as relates to capacitation
[28], is related to the time needed to reach the concentration of $\mathrm{NO}$ appropriate for capacitation and the duration of time spent at this concentration of NO, rather than the concentration itself. These results are in accordance with the findings of du Plessis et al. [29], which also provided evidence that the degree of NO concentration has no significant correlation to semen quality parameters in sperm capacitation.

Several studies have examined the manipulation of NO concentration and its effect on sperm function. Nitric oxide donors have been shown to improve overall sperm quality [30]. Sodium nitroprusside has been shown to

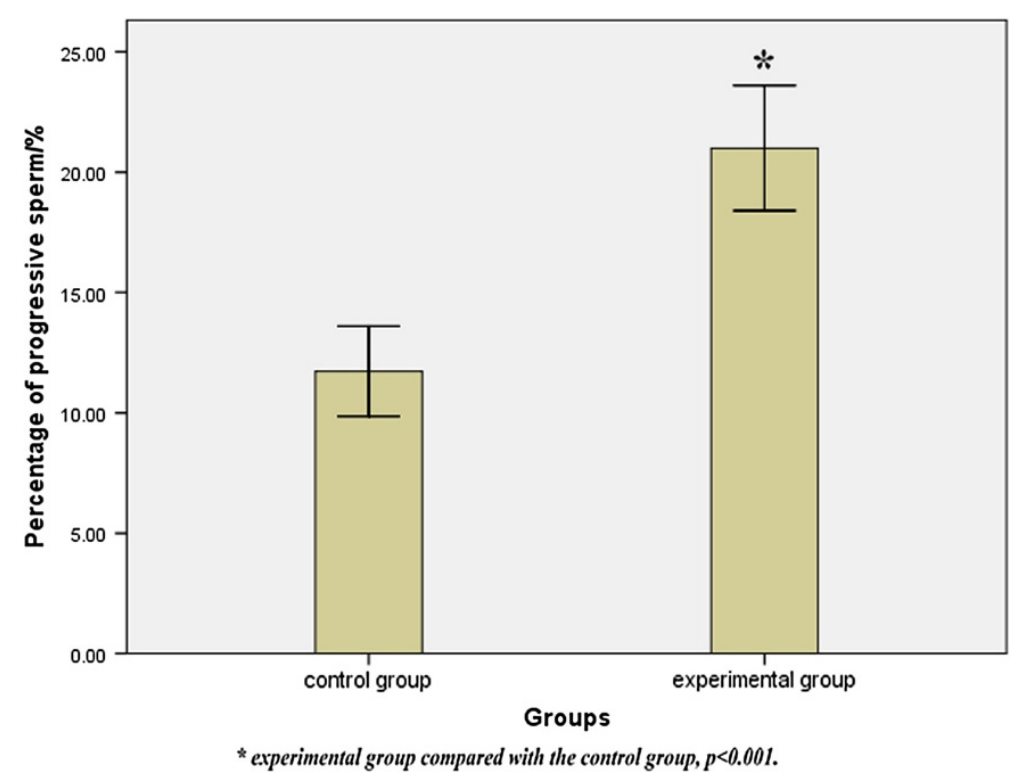

Figure 7 Percentage of progressive sperm in the control and experimental groups. Results were expressed as mean $\pm S D, n=15$. There was a statistically significant difference between the control and experimental groups in the percentage of progressive sperm $(t=-6.201, p<0.001)$. 
improve post-thaw sperm motility [31], improve motility and viability [32], inhibit sperm membrane oxidative damage [31,32], and increase intracellular cGMP in both normal and asthenozoospermic semen samples [32]. Nitric oxide donors have also been shown to directly simulate the acrosome reaction in mouse sperm [33]. In other animal models, inhibition of NO synthesis decreases sperm motility and reduces hyperactivation in the late stage of capacitation [34], and inhibits the acrosome reaction [35].

Our present study provides further support for the effects of NO concentration of sperm function. Based on our results, we speculate that the sperm function of the abnormal semen group is affected by two factors: the delay in reaching an appropriate NO concentration in the early stage of sperm capacitation in vitro, and the extended duration of time spent at this NO concentration in the late stage of sperm capacitation in vitro. In addition, the persistence of $\mathrm{NO}$ appears to influence sperm viability, likely because the activation of NO-related enzymes in abnormal semen is slow than in normal semen, causing the sperm to be exposed to a certain NO concentration for a longer time, a condition that could have a detrimental effect on sperm progression and sperm capacitation in vitro. By manipulating $\mathrm{NO}$ concentration in the experimental abnormal sperm group (C2) using SNP and L-NMMA, we were able to maintain the NO concentration at a constant level, similar to that of the normal semen group. We were also able to reproduce a time change curve of NO concentration similar to that of the normal semen group, and in particular, were able to approximate the NO concentration of late stage sperm capacitation in vitro. Using this process, we were able to demonstrate a significant increase in the percentage of progressive sperm, and show that by controlling the NO concentration and time duration, we can improve both sperm motility and sperm capacitation in vitro. These findings suggest that clinical utilization of NO-related preparations may improve sperm motility and function, and thus conception rates, in infertile and subfertile men.

\section{Conclusions}

By studying the mechanism of action of NO in sperm capacitation, we recognize there is certain regularity to both NO concentration and its time change in regards to sperm motility in vitro, and that these factors are conducive to capacitation. Based on our current results, further research into developing preparations of $\mathrm{NO}$ that can be used with in vitro fertilization to improve both sperm quality and fertilization rates may be considered. However, further basic work remains to be done to provide additional evidence to support the use of $\mathrm{NO}$ preparations in this capacity. Our experiment also has some limitations affecting the outcomes, such as the most effective timing and dosing of NO-related preparations, and the effects of the differences of the sperm capacitation process in vitro and in vivo. What we found from our present study are only preliminary results, and the safety of improving the sperm motility through regulation of the NO concentration should be further confirmed. While work in this area is quite complicated in both design and execution, we believe that a more detailed understanding of the action of NO will provide the basis for significant advances in the clinical treatment of male factor infertility.

\section{Methods \\ Materials}

Semen samples were obtained from the infertility clinic and outpatient urology clinic of the Second Affiliated Hospital in Quanzhou, Fujian, China. All participants were randomly selected and signed informed consent for the study. The normal semen samples $(n=15)$ were from men aged 23 to 31 years old (mean 25.5 years), and the abnormal semen samples $(n=15)$ were from men aged 24 to 30 years old (mean 24.9 years). Classification of the samples to the normal or abnormal groups conformed to the World Health Organization (WHO) semen testing standards [36]. Criteria for the normal group included semen liquefaction time $<30$ minutes, sperm concentration $\geq 15 \times 10^{6} / \mathrm{ml}$, sperm viability $\geq 58 \%$, and percentage of progressive sperm $\geq 32 \%$. Criteria for the abnormal sperm group included semen liquefaction time $>30$ minutes, sperm concentration $<15 \times 10^{6} / \mathrm{ml}$, sperm viability $<58 \%$, and percentage of progressive sperm $<32 \%$.

\section{Semen analysis and sperm processing}

Subjects were instructed to abstain from ejaculation for 3 to 5 days prior to sample collection. Semen samples were obtained via masturbation, collected in disposable sterile containers, and kept at room temperature until liquefaction occurred. Semen analysis was completed using a computer-assisted semen analyzer. Sperm were isolated from semen samples by using Percoll gradient centrifugation [37]. Sperm samples were incubated at $37^{\circ} \mathrm{C}$ for one hour in Earle's balanced salt solution (SigmaAldrich, St. Louis, MO) [36,38] before utilization.

\section{Nitric oxide detection}

$\mathrm{NO}$ detection was carried out using the inNO-T nitric oxide measurement system consisting of an $\mathrm{NO}$ sensor and inNO System (Innovative Instruments, Inc., Tampa, $\mathrm{FL}$ ), allowing the NO concentration to be measured by the NO sensor, with numeric values displayed simultaneously. The NO sensor was calibrated before each experiment. Our study group had successfully used 
this system previously to monitor the concentration of NO [39].

\section{Experimental procedures}

We chose semen samples for the normal sperm group (group $\mathrm{A}, \mathrm{n}=15$ ) and the abnormal sperm group (group $B, n=15)$ based on initial semen analysis. Sperm were obtained via Percoll gradient centrifugation and reached capacitation via sperm culture as described earlier. Changes in NO concentration during sperm capacitation were measured in real time for both groups A and B.

An additional group of abnormal semen samples (group $\mathrm{C}, \mathrm{n}=30$ ) was randomly selected and divided into a control (C1) and an experimental (C2) group. Sperm processing methods were the same as with groups $\mathrm{A}$ and $\mathrm{B}$, and NO concentration and time changes were measured in vitro while manipulating $\mathrm{NO}$ concentration. Thirty minutes after starting measurement of $\mathrm{NO}$ concentration (the average time of normal sperm capacitation in vitro), SNP $(100 \mathrm{nmol} / \mathrm{L})$ was continuously added to group C2, with a subsequent rise in and stabilization of $\mathrm{NO}$ concentration at around 19,000 nM-20,000 nM. At forty-one minutes (the average duration of time for normal sperm capacitation in vitro), L-NMMA (10 nmol/L) was added to group $\mathrm{C} 2$ until changes in the $\mathrm{NO}$ concentration returned to baseline. During the procedures, NO-related preparations were placed into containers connected with the infusion tube, and then the preparations were added at a constant rate, controlled by the regulating valve on the infusion tube. AmiNO-700 sensors were placed into the sperm samples, measuring the change of the NO concentration with an associated curve shown on the analyzer computer. The inNO-T nitric oxide measurement system was used for the in situ detection and real time measurement of NO. Sperm quality parameters were measured for both groups.

\section{Statistical analysis}

We performed statistical analysis using SPSS 17.0 (IMB, Armonk, NY). Results are shown as mean \pm standard deviation. Data correlation analysis was performed between groups, and in-group data were analyzed using the independent sample t-test and Pearson correlation analysis. We considered results to be significant at $p<0.05$.

\footnotetext{
Abbreviations

ROS: Reactive oxygen species; $\mathrm{H}_{2} \mathrm{O}_{2}$ : Hydrogen peroxide; NOS: Nitric oxide synthase; NO: Nitric oxide; SNP: Sodium nitroprusside; L-NMMA: NG-Monomethyl-L-arginine; $T 1$ : The basal time (the time needed to reach the appropriate concentration of NO); T2: The duration of time (at the appropriate NO concentration); D: The concentration of NO; C1: The control group, C2: the experimental group.
}

\section{Competing interests}

The authors declare that they have no competing interests.

\section{Authors' contributions}

JW carried out the semen analyses and sperm processing, NO detection, and drafted the manuscript. $\mathrm{QH}$ participated in the experimental procedures, performed the data analysis and interpretation of data, and drafted the manuscript. XY conceived the study and its design, and helped revise the manuscript. YC contributed to the study's design and assisted with statistical analysis. JC assisted with acquisition of data and with revision of the manuscript. All authors read and approved the final manuscript. As joint first authors, both JW and QH contributed equally to the research work.

\section{Acknowledgments}

This project was supported by the Health Bureau of Quanzhou, Fujian, China. The authors would like to express their sincere thanks to the reviewers and Editor-in-Chief of the journal for their valuable comments and suggestions. We appreciate the assistance provided by the facilities of the Department of Urology, infertility clinic and clinical laboratory at the Second Affiliated Hospital of Fujian Medical University. Finally, we would like to thank Kevin Vassar for his assistance with the background section and with general English translation.

\section{Author details}

${ }^{1}$ Departments of Urology, Affiliated Hospital, Shandong Medical college, Linyi, Shandong, China. 'Departments of Urology, Second Affiliated Hospital, Fujian Medical University, Quanzhou, Fujian, China. ${ }^{3}$ Departments of Ophthalmology, Second Affiliated Hospital, Fujian Medical University, Quanzhou, Fujian, China.

Received: 3 March 2014 Accepted: 27 August 2014

Published: 18 September 2014

\section{References}

1. Male Infertility Best Practice Statement Panel: The Optimal Evaluation of the Infertile Male: AUA Best Practice Statement. Linthicum, Maryland: American Urological Association; 2010.

2. De Lamirande $E$, O'Flaherty C: Sperm activation: role of reactive oxygen species and kinases. Biochim Biophys Acta 2008, 1784:106-115.

3. Pourova J, Kottova M, Voprsalova M, Pour M: Reactive oxygen and nitrogen species in normal physiological processes. Acta Physiol 2010, 198:15-35.

4. Moncada S, Palmer RMJ, Higgs EA: Nitric oxide: physiology, pathophysiology, and pharmacology. Pharmacol Rev 1991, 43:109-142.

5. Rosselli M, Keller PJ, Dubey RK: Role of nitric oxide in the biology, physiology and pathophysiology of reproduction. Hum Reprod Update 1998, 4:3-24.

6. Doshi SB, Khullar K, Sharma RK, Agarwal A: Role of reactive nitrogen species in male infertility. Reprod Biol Endocrinol 2012, 10:109.

7. Zini A, De Lamirande E, Gagnon C: Low levels of nitric oxide promote human sperm capacitation in vitro. J Androl 1995, 16:424-431.

8. Herrero MB, De Lamirande E, Gagnon C: Nitric oxide is a signaling molecule in spermatozoa. Curr Pharm Des 2003, 9:419-425.

9. Aitken RJ, Baker MA, De luliis GN, Nixon B: New insights into sperm physiology and pathology. In Fertility Control. Edited by Habenich UF, Aitken RJ. Berlin, Germany: Springer-Verlag; 2010:99-115.

10. Zini A, O'Bryan MK, Magid MS, Schlegel PN: Immunohistochemical localization of endothelial nitric oxide synthase in human testis, epididymis, and vas deferens suggests a possible role for nitric oxide in spermatogenesis, sperm maturation, and programmed cell death. Biol Reprod 1996, 55:935-941.

11. Roessner C, Paasch U, Glander HJ, Grunewald S: Activity of nitric oxide synthase in mature and immature human spermatozoa. Andrologia 2010, 42:132-137.

12. Revelli A, Soldati G, Costamagna C, Pellery O, Aldieri E, Massobrio M, Bosia A, Ghigo D: Follicular fluid proteins stimulate nitric oxide (NO) synthesis in human sperm: a possible role for NO in acrosomal reaction. J Cell Physiol 1999, 178:85-92.

13. Lewis SEM, Donnelly ET, Sterling ESL, Kennedy MS, Thompson W, Chakravarthy U: Nitric oxide synthase and nitrite production in human spermatozoa: evidence that endogenous nitric oxide is beneficial to sperm motility. Mol Hum Reprod 1996, 2:873-878.

14. Revelli A, Bergandi L, Massobrio M, Lindblom B, Bosia A, Ghigo D: The concentration of nitrite in seminal plasma does not correlate with sperm concentration, sperm motility, leukocytospermia, or sperm culture. Fertil Steril 2001, 76:496-500. 
15. Salvolini E, Buldreghini E, Lucarini G, Vignini A, Di Primio R, Balercia $G$ : Nitric oxide synthase and tyrosine nitration in idiopathic asthenozoospermia: an immunohistochemical study. Fertil Steril 2012, 97:554-560.

16. Weinberg JB, Doty E, Bonaventura J, Haney AF: Nitric oxide inhibition of human sperm motility. Fertil Steril 1995, 64:408-413.

17. Rosselli M, Dubey RK, Imthurn B, Macas E, Keller PJ: Effects of nitric oxide on human spermatozoa: evidence that nitric oxide decreases sperm motility and induces sperm toxicity. Hum Reprod 1995, 10:1786-1790.

18. Nobunaga T, Tokugawa Y, Hashimoto K, Kubota Y, Sawai K, Kimura T, Shimoya K, Takemura M, Matsuzaki N, Azuma C, Saji F: Elevated nitric oxide concentration in the seminal plasma of infertile males: nitric oxide inhibits sperm motility. Am J Reprod Immunol 1996, 36:193-197.

19. O'Bryan MK, Zini A, Cheng CY, Schlegel PN: Human sperm endothelial nitric oxide synthase expression: correlation with sperm motility. Fertil Steril 1998, 70:1143-1147.

20. Wu TP, Huang BM, Tsai HC, Lui MC, Liu MY: Effects of nitric oxide on human spermatozoa activity, fertilization and mouse embryonic development. Arch Androl 2004, 50:173-179.

21. Balercia G, Moretti S, Vignini A, Magagnini M, Mantero F, Boscaro M, Ricciardo-Lamonica G, Mazzanti L: Role of nitric oxide concentrations on human sperm motility. J Androl 2004, 25:245-249.

22. Du Plessis SS, McAllister DA, Luu A, Savia J, Agarwal A, Lampiao F: Effects of $\mathrm{H}_{2} \mathrm{O}_{2}$ exposure on human sperm motility parameters, reactive oxygen species levels and nitric oxide levels. Andrologia 2010, 42:206-210.

23. Ramya T, Misro MM, Sinha D, Nandan D, Mithal S: Altered levels of seminal nitric oxide, nitric oxide synthase, and enzymatic antioxidants and their association with sperm function in infertile subjects. Fertil Steril 2011, 95:135-140.

24. Herrero MB, Gagnon C: Nitric oxide: a novel mediator of sperm function. J Androl 2001, 22:349-356.

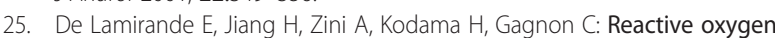
species and sperm physiology. Rev Reprod 1997, 2:48-54.

26. Ford WCL: Regulation of sperm function by reactive oxygen species. Hum Reprod Update 2004, 10:387-399.

27. De Lamirande E, Lamothe G, Villemure M: Control of superoxide and nitric oxide formation during human sperm capacitation. Free Rad Biol Med 2009, 46:1420-1427.

28. Thundahill J, De Lamirande E, Gagnon C: Nitric oxide regulates the phosphorylation of the threonine-glutamine-tyrosine motif in proteins of human spermatozoa during capacitation. Biol Reprod 2003, 68:1291-1298.

29. Du Plessis SS, McAllister DA, Luu A, Savia J, Agarwal A, Lampiao F: Effects of $\mathrm{H}_{2} \mathrm{O}_{2}$ on human sperm motility parameters, reactive oxygen species levels and nitric oxide levels. Andrologia 2010, 42:206-210.

30. Leal AC, Caldas-Bussiere MC, Carvalho CS, Viana KS, Quirino CR: Role of nitric oxide on quality of freshly ejaculated bull spermatozoa during heparin-induced in vitro capacitation. Anim Reprod Sci 2009, 116:38-49.

31. Hellstrom WJ, Bell M, Wang R, Sikka SC: Effect of sodium nitroprusside on sperm motility, viability, and lipid peroxidation. Fertil Steril 1994, 61:1117-1122.

32. Zhang $H$, Zheng RL: Possible role of nitric oxide on fertile and asthenozoospermic infertile human sperm function. Free Rad Res 1996, 25:347-354.

33. Herrero MB, Viggiano JM, Perez Martinez S, De Gimeno MF: Evidence that nitric oxide synthase is involved in progesterone induced acrosomal exocytosis in mouse spermatozoa. Reprod Fertil Dev 1997, 9:433-439.

34. Yeoman RR, Jones WD, Rizk BM: Evidence for nitric oxide regulation of hamster sperm hyperactivation. J Androl 1998, 19:58-64.

35. Viggiano JM, Herrero MB, Martinez SP, De Gimeno MF: Analysis of the effect of nitric oxide synthase inhibition on mouse sperm employing a modified staining method for assessment of the acrosome reaction. J Androl 1996, 17:692-698.

36. World Health Organization: WHO Laboratory Manual for the Examination and Processing of Human Semen. 5th edition. Geneva: WHO; 2010.

37. van der Zwalmen P, Bertin-Segal G, Geerts L, Debauche C, Schoysman R: Sperm morphology and IVF pregnancy rate: comparison between Percoll gradient centrifugation and swim-up procedures. Hum Reprod 1991, 6:581-588.
38. Yang MG, Yang Y, Huang P, Hao XK, Zhang ZY, Zheng SL, Fan AL, Rao GZ, Wei XM: Sodium nitroprusside facilitates human sperm capacitation and acrosome reaction [in Chinese]. Zhonghua Nan Ke Xue 2005, 11:422-425.

39. Xingyu Y, Guoliang T, Youmei C, Honggen W, Yihong G, Junyi C: The effect of nitric oxide on the pressure of the acutely obstructed ureter. Urol Res 2012, 40:163-169.

doi:10.1186/0717-6287-47-44

Cite this article as: Wang et al.: Effect of exogenous nitric oxide on sperm motility in vitro. Biological Research 2014 47:44.

\section{Submit your next manuscript to BioMed Central and take full advantage of:}

- Convenient online submission

- Thorough peer review

- No space constraints or color figure charges

- Immediate publication on acceptance

- Inclusion in PubMed, CAS, Scopus and Google Scholar

- Research which is freely available for redistribution

Submit your manuscript at www.biomedcentral.com/submit
C) BioMed Central 